\title{
DESIGN OF A NOVEL HAND-HELD PNEUMATIC VACUUM PAD USING CFD
}

Jyh-Chyang Renn

Department of Mechanical Engineering, National Yunlin University of Science and Technology, 640 Douliou, Taiwan, R.O.C., rennjc@yuntech.edu.tw

\section{Chin-Yi Cheng}

Department of Mechanical Engineering, National Yunlin University of Science and Technology, 640 Douliou, Taiwan, R.O.C.

Yong-Chao Yang

Department of Mechanical Engineering, National Yunlin University of Science and Technology, 640 Douliou, Taiwan, R.O.C.

Follow this and additional works at: https://jmstt.ntou.edu.tw/journal

Part of the Engineering Commons

\section{Recommended Citation}

Renn, Jyh-Chyang; Cheng, Chin-Yi; and Yang, Yong-Chao (2010) "DESIGN OF A NOVEL HAND-HELD PNEUMATIC VACUUM PAD USING CFD," Journal of Marine Science and Technology. Vol. 18: Iss. 5, Article 2.

DOI: $10.51400 / 2709-6998.1900$

Available at: https://jmstt.ntou.edu.tw/journal/vol18/iss5/2

This Research Article is brought to you for free and open access by Journal of Marine Science and Technology. It has been accepted for inclusion in Journal of Marine Science and Technology by an authorized editor of Journal of Marine Science and Technology. 


\section{DESIGN OF A NOVEL HAND-HELD PNEUMATIC VACUUM PAD USING CFD}

Acknowledgements

The financial support of the National Science Council under grant number NSC-97-2622-E-224-006-CC3 is greatly appreciated. 


\title{
DESIGN OF A NOVEL HAND-HELD PNEUMATIC VACUUM PAD USING CFD
}

\author{
Jyh-Chyang Renn*, Chin-Yi Cheng*, and Yong-Chao Yang*
}

Key words: pneumatics, CFD, vacuum pad, automation.

\begin{abstract}
In this paper, a novel hand-held pneumatic vacuum pad is developed using CFD-simulation. CFD is the abbreviation of Computational Fluid Dynamics, which is a powerful and reliable tool to provide an effective insight into the flow field numerically. Nowadays, application of pneumatic vacuum pads may be found in many different real industries, especially in the field of automatic conveyer system, automatic or manual assembly line, semiconductor industry as well as wafer factory, etc. Traditional vacuum pads are generally mounted on robots or other machines to form automated manufacturing systems. In this paper, however, a novel cylindrical vacuum pad specially designed for the easy grasp by the operator's hand is proposed. To achieve two preset requirements, that is, large lifting-force output and small dimension, the commercial CFDRC software is utilized as a tool to simulate the airflow field and obtain the most suitable geometry for the vacuum pad. Finally, experimental results further prove that the new vacuum pad with two aforementioned features is successfully developed and realized.
\end{abstract}

\section{INTRODUCTION}

Nowadays, vacuum technology is widely applied in many different industries, especially in the field of vacuum packing machinery, clean room technology, semiconductor manufacturing process and high-precision measurement devices like the mass spectrometer, SEM, TEM, etc. In these applications, vacuum pump is generally utilized to create the effect of vacuum $[2,6,12]$. In this paper, however, a simple, cylindrical hand-held pneumatic vacuum pad is proposed and developed by using CFD-simulation [10]. Generally speaking, CFD is widely used in different areas concerning fluid flow, heat transfer, etc. In the modern fluid power technology, however, some recent papers show that CFD is proven to be a successful approach to analyze and compensate the flow induced flow force

Paper submitted 02/06/09; revised 08/01/09; accepted 08/29/09. Author for correspondence: Jyh-Chyang Renn (e-mail: rennjc@yuntech.edu.tw).

*Department of Mechanical Engineering, National Yunlin University of Science and Technology, 640 Douliou, Taiwan, R.O.C. inside the valve body $[4,10,14]$. Unlike the vacuum pump, the vacuum generating principle of the newly developed vacuum pad is actually based on the well-known Bernoulli's equation. The Bernoulli's equation states that the fluid pressure will decrease if the velocity of fluid flow is increased. In a traditional vacuum pad, as shown in Fig. 1, a nozzle is generally utilized to increase the fluid flow velocity and hence to produce the effect of vacuum [13]. In this paper, however, a similar but much simplified vacuum pad is proposed as shown in Fig. 2. It is observed that, instead of the nozzle, a sudden

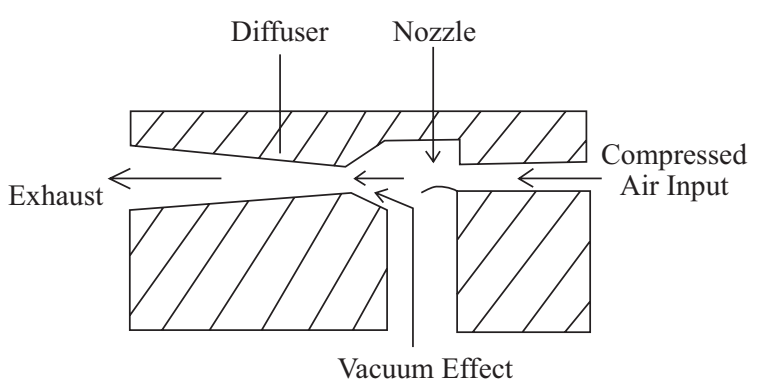

Fig. 1. Scheme of a traditional vacuum pad using nozzle to produce vacuum [5].

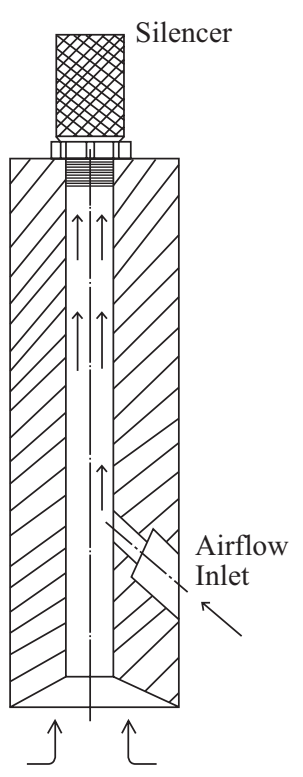

Fig. 2. Scheme of the newly developed hand-held pneumatic vacuum pad. 


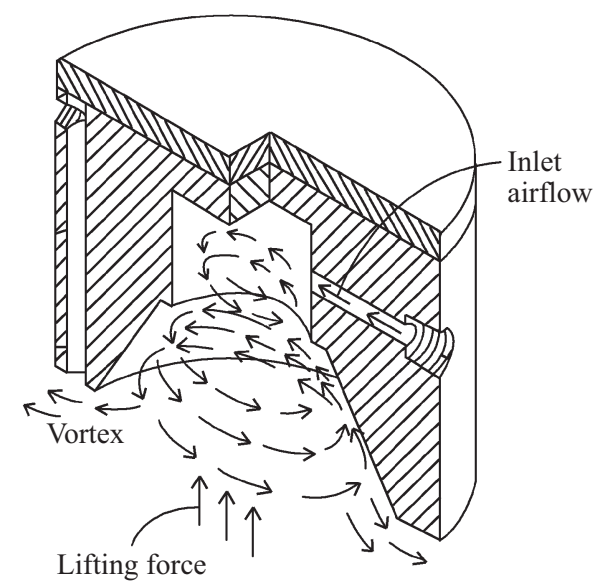

Fig. 3. New principle of vacuum generation - Vortex flow field.

reduction in the cross section of the pipe is designed to accelerate the fluid flow. Therefore, two major features are integrated into the design. The first feature is the cylindrical body design enabling the easy grasp by the operator's hand. In addition, compared to the complex profile of the nozzle, the inexpensive manufacturing arising from the very simple geometric design of sudden reduction in the pipe cross section is the second feature. As mentioned previously, the traditional design using nozzles dominates the market for quite a long time. However, one brand new vacuum generation principle was proposed in recent two years. In details, the so-called vortex flow field theory that can be used to construct non-contact (floating) or contact vacuum pads was presented by the author [7-9]. As shown in Fig. 3, the air is guided to flow through an eccentric inlet port and flow out through the outlet between the vacuum pad and the lifted work-piece. Similar to the structure of a tropical typhoon, the eccentric inlet port generates a vortex flow field, which accelerates the rotational airflow. Accordingly, the air pressure near the center line of the vacuum pad decreases because of the increased airflow velocity. Consequently, the effect of vacuum is generated. Almost at the same time, Prof. Kagawa also proposed the same theory and applied it successfully to the design of small-sized vacuum pad for picking up silicon wafers [5]. One minor fault of the new floating vacuum pad, however, is that the lifted wafer or other work-piece may shift or even rotate. To overcome such a disadvantage, four or six small-sized vacuum pads are symmetrically assembled together to form a round pick-up device for silicon wafer, TFT or solar energy panels. The scheme is shown in Fig. 4. On the other hand, the fundamental comparison between floating as well as contact vacuum pads is described as follows. The former is suitable for some precision pick-up applications, like the pick-up devices for silicon wafer, TFT or solar energy panel, because it will not damage the surface of the lifted wafer or panel [5, 7-9]. Compared to floating vacuum pads, the developed vacuum pad is actually based on the contact principle. It means that the lifted workpiece contacts the bottom of the pad while the lifting force is

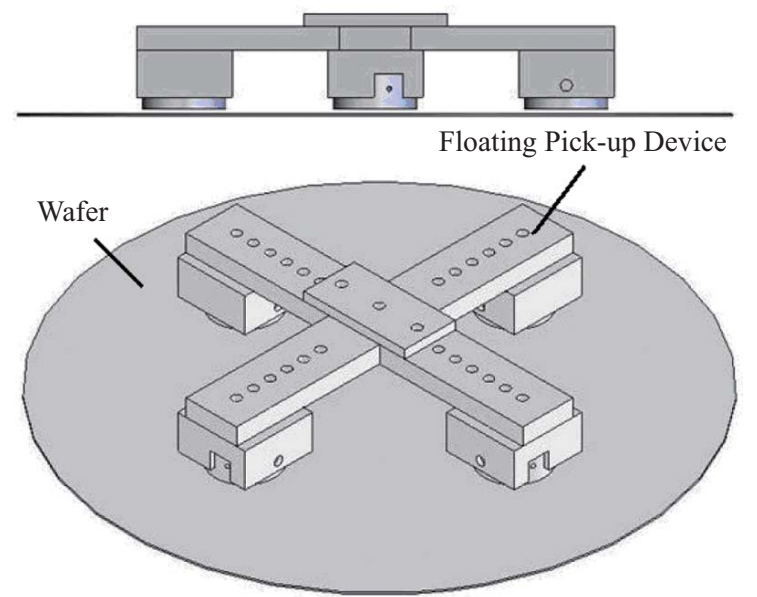

Fig. 4. Round floating pick-up device for silicon wafer, TFT and solar-energy panels.

generated. Such a design prevents the possible rotation and shifting of the work-piece $[11,13]$. Typical application fields of such a pneumatic vacuum pad include the factory automation system, automatic or manual assembly line and conveyer system, etc. [3].

To fulfill two preset requirements, that is, large lifting-force output and small dimension, the commercial CFD-RC software is utilized as a tool to simulate the air flow field and obtain the most suitable geometrical dimensions for the vacuum pad. After some tedious trial-and-errors, a simple hand-held pneumatic vacuum pad is successfully developed and a corresponding prototype is also produced. To test the static performance of the new vacuum pad, a test bench is constructed. In the following, the design of the vacuum pad using CFD-simulation is firstly illustrated in details.

\section{DESIGNING THE PNEUMATIC VACUUM PAD BY CFD-SIMULATION}

First of all, the basic principle of applying Bernoulli's equation to the design of the hand-held pneumatic vacuum pad is described as follows. Bernoulli's equation can be derived from the energy conservation law and its general form using the expression head is written as [10]

$$
\frac{V_{1}^{2}}{2 g}+\frac{P_{1}}{W}+Z_{1}=\frac{V_{2}^{2}}{2 g}+\frac{P_{2}}{W}+Z_{2},
$$

where $V_{1}$ : velocity of fluid at station 1 ,

$P_{1}$ : pressure of fluid at station 1 ,

$Z_{1}$ : elevation head at station 1 ,

$V_{2}$ : velocity of fluid at station 2 ,

$P_{2}$ : pressure of fluid at station 2 ,

$Z_{2}$ : elevation head at station 2 ,

$g$ : gravity acceleration,

$W$ : weight density of fluid. 


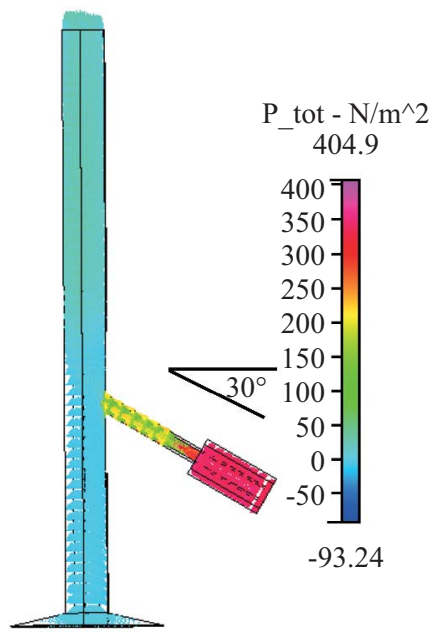

Fig. 5. Simulation result of the pressure distribution. (inlet airflow angle $=\mathbf{3 0}{ }^{\circ}$ ).

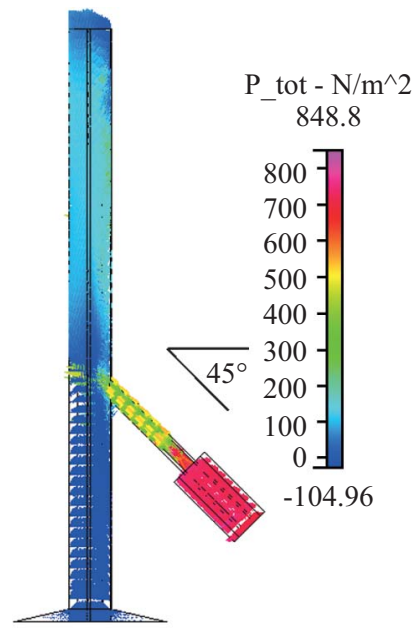

Fig. 6. Simulation result of the pressure distribution. (inlet airflow angle $=45^{\circ}$ ).

the vacuum pad, a hot-wire anemometer is utilized.

(2) The airflow is compressible.

(3) The initial air density is assumed to be $1.189 \mathrm{~kg} / \mathrm{m}^{3}$ at room temperature. In the real CFD-simulation, however, the mode of airflow is chosen to be compressible. That is, only the initial density of air is given and the density of air may change with temperature and pressure to agree with the real situation.

(4) The dynamic viscosity of the air is set to be $1.789 \times 10^{-5}$ $\mathrm{N} \cdot \mathrm{s} / \mathrm{m}^{2}$ at room temperature.

(5) The room temperature is set to be $20^{\circ} \mathrm{C}$ or $293^{\circ} \mathrm{K}$.

(6) The reference acoustic velocity of air is assumed to be 340 $\mathrm{m} / \mathrm{s}$.

(7) The boundaries of the flow field are considered as the wall, which means that no airflow across the boundaries is allowed.

(8) No heat transfer and chemical reaction exists in the flow field.

(9) The outlet conditions are set to be atmospheric pressure and room temperature.

Figures 5-7 show the corresponding simulation results of pressure distribution for three different inlet airflow angles respectively, in which the cone-shaped angle is temporarily set to be $15^{\circ}$. The graphical comparison of the derived gauge pressures is shown in Fig. 8. It is observed that the design with inlet airflow angle of $60^{\circ}$ possesses the lowest pressure near the center line of the vacuum pad. Among three different angles, therefore, it is clear that the most suitable inlet airflow angle is $60^{\circ}$. Similarly, three different engineering angles $\left(45^{\circ}\right.$, $30^{\circ}$ and $15^{\circ}$ ) for the cone-shaped space at the bottom of the vacuum pad are also chosen for CFD-simulations as shown in Fig. 9. In these simulations, the inlet airflow angle is set to be $60^{\circ}$. After comparing the derived vacuum gauge pressure shown in Fig. 10, it is observed that the lowest pressure near the center line of the vacuum pad occurs for the cone-shaped 


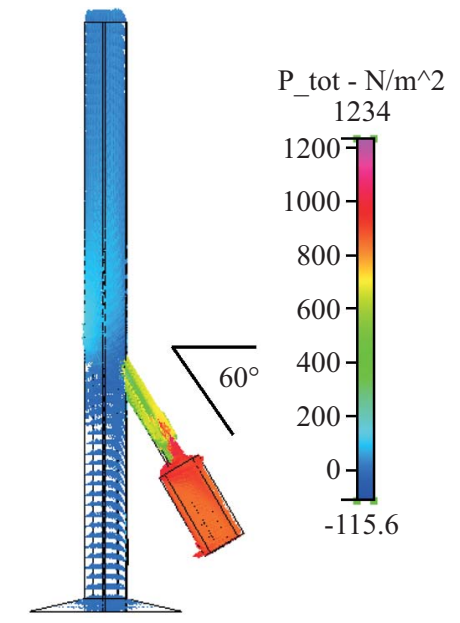

Fig. 7. Simulation result of the pressure distribution. (inlet airflow angle $=60^{\circ}$ ).

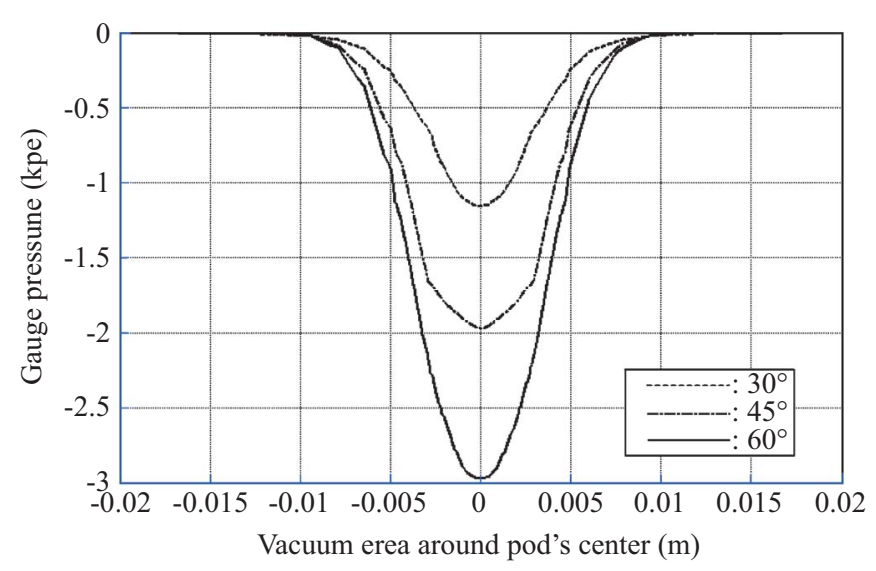

Fig. 8. Graphical comparison of the gauge pressure for three different inlet airflow angles.

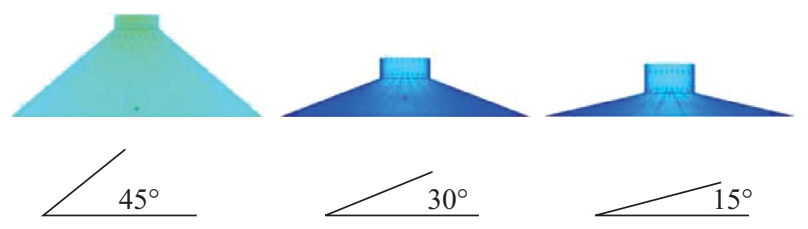

Fig. 9. Three different angles for the cone-shaped space at the bottom of the vacuum pad.

angle of $15^{\circ}$. Thus, the angle of $15^{\circ}$ is chosen for the design. It is worth mentioning that the three dimensions shown in Fig. 9 have the same effective area of pressure actuation. Therefore, as depicted in Fig. 11, the generated lifting force would be

$$
F_{\text {lift }}=A\left(P_{a t m}-P_{v}\right)=-A_{v} \cdot P_{v}
$$

where $F_{\text {lift }}$ : lifting force output,

$A_{v}$ : effective area of pressure actuation,

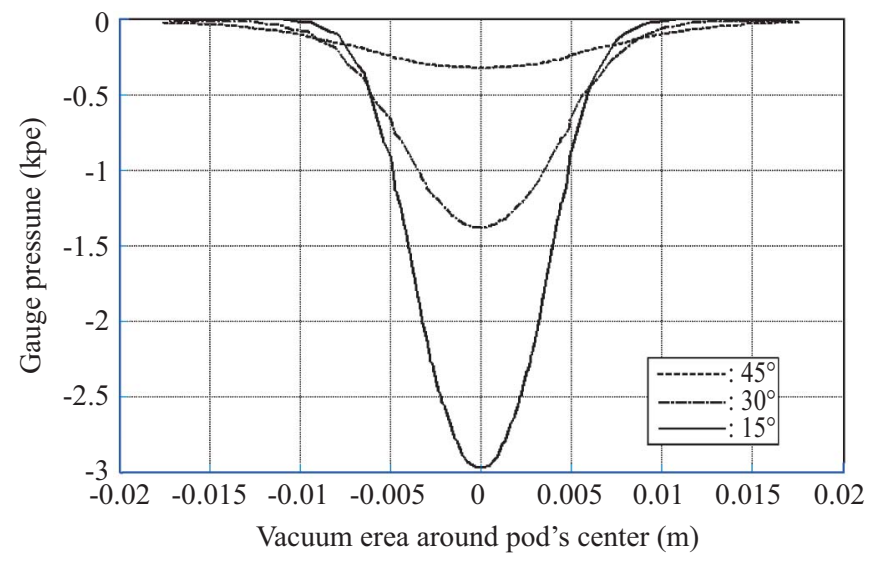

Fig. 10. Graphical comparison of the gauge pressure for three different cone-shaped angles.

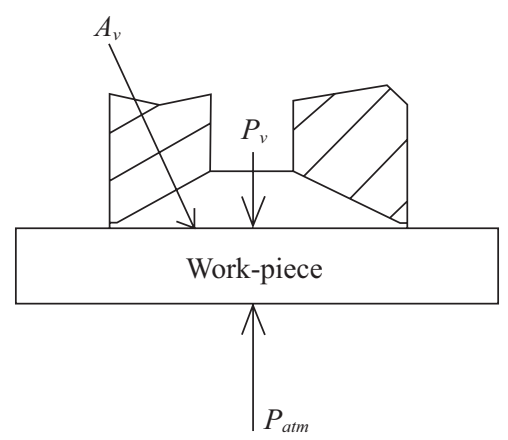

Fig. 11. Scheme showing the calculation of lifting force output.

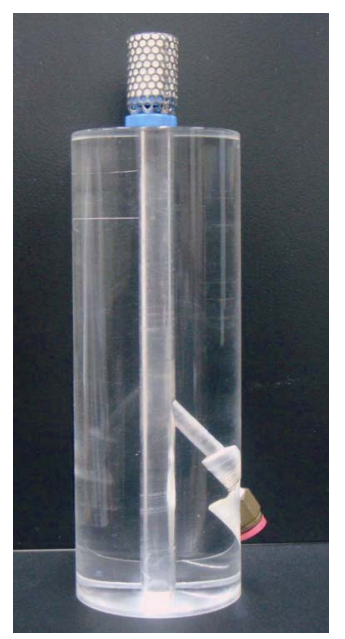

Fig. 12. Prototype of the developed hand-held vacuum pad.

$P_{\text {atm }}:$ atmospheric gauge pressure $\left(P_{\text {atm }}=0 \mathrm{kPa}\right)$, $P_{v}$ : vacuum gauge pressure (negative value).

It can further be concluded that the lifting force output would be larger if the vacuum gauge pressure is lower. Finally, the picture of the manufactured prototype is shown in Fig. 12. 


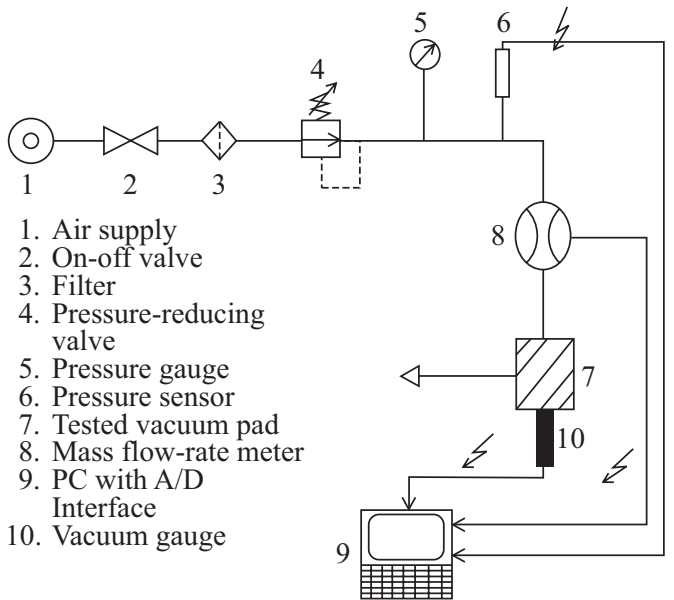

Fig. 13. Layout of the test device for pneumatic vacuum pad.

\section{DESIGN OF TEST DEVICE}

To test the static performance of the developed pneumatic vacuum pad, a test device is constructed to measure the vacuum pressure at the center of the vacuum pad. The circuit layout of the entire test device is shown in Fig. 13. The supply air pressure is fixed at 6 bar and a pressure-reducing valve is utilized to adjust the input pressure to the vacuum pad. Figure 14 shows the scheme for measuring the vacuum pressure. In details, a vacuum pressure sensor (SMC, ZSE30/ISE30) is inserted into the work-piece, so that the vacuum pressure at the center of the vacuum pad can be measured.

\section{RESULTS AND DISCUSSIONS}

In this section, both simulation and experimental results concerning the developed vacuum pad are summarized and discussed. Firstly, Table 1 shows different values of the vacuum pressure at the center of the vacuum pad derived by simulation and experiment respectively. It is also noticeable that the vacuum pressure is represented by the gauge pressure, which is actually a negative value. The inlet pressure to the vacuum pad is varied from 1 bar to 5 bar by adjusting the pressure reducing valve. The initial input air velocity for the CFDsimulation is set to be $6 \mathrm{~m} / \mathrm{s}$ for the input pressure of $1 \mathrm{bar}$, $17 \mathrm{~m} / \mathrm{s}$ for $3 \mathrm{bar}, 22 \mathrm{~m} / \mathrm{s}$ for 4 bar and $28 \mathrm{~m} / \mathrm{s}$ for 5 bar, respectively. After calculations, the maximal deviation between simulation and experimental result for different supply air pressure is found to be in the range of $7 \%$ to $28 \%$. In details, as shown in Fig. 15, the experimental lifting force without silencer agrees quite well with the simulation result. The error is below $5 \%$. However, the deviation between the experimental lifting force with a silencer and the simulation result is dependent on the supply pressure. For the supply pressure of 1 bar, the error is around $7 \%$. However, the percentage error increases to $28 \%$ if the supply pressure is set to be 5 bar. One possible reason for that is the larger airflow resistance through the silencer if the airflow velocity is higher due to larger supply air pressure.

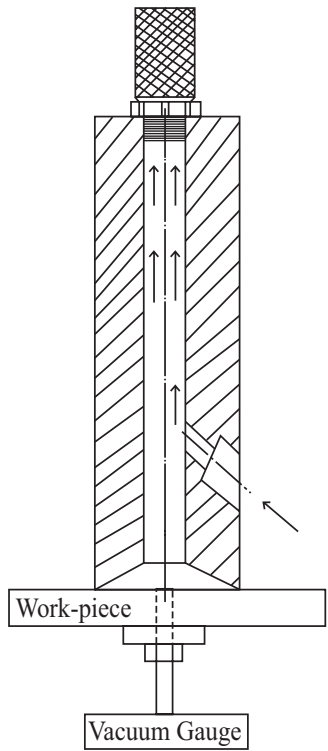

Fig. 14. Scheme of test device for measuring vacuum pressure.

Table 1. Simulation and experimental results of vacuum pressure at the center of the vacuum pad.

\begin{tabular}{|c|c|c|c|}
\hline Airsupply pressure & Experimental and \\
simulation values & $\begin{array}{c}\text { CFD simulation } \\
\text { vacuum pressure }\end{array}$ & $\begin{array}{c}\text { Experimental } \\
\text { vacuum pressure } \\
\text { (without silencer) }\end{array}$ & $\begin{array}{c}\text { Experimental } \\
\text { vacuum pressure } \\
\text { (with a silencer) }\end{array}$ \\
\hline $1.0 \mathrm{bar}$ & $-1.0 \mathrm{kpa}$ & $-1.1 \mathrm{kpa}$ & $-0.94 \mathrm{kpa}$ \\
\hline $1.5 \mathrm{bar}$ & $-1.7 \mathrm{kpa}$ & $-1.7 \mathrm{kpa}$ & $-1.4 \mathrm{kpa}$ \\
\hline $2.0 \mathrm{bar}$ & $-2.9 \mathrm{kpa}$ & $-2.8 \mathrm{kpa}$ & $-2.3 \mathrm{kpa}$ \\
\hline $2.5 \mathrm{bar}$ & $-3.8 \mathrm{kpa}$ & $-4.0 \mathrm{kpa}$ & $-3.3 \mathrm{kpa}$ \\
\hline $3.0 \mathrm{bar}$ & $-5.3 \mathrm{Kpa}$ & $-5.0 \mathrm{kpa}$ & $-4.1 \mathrm{kpa}$ \\
\hline $3.5 \mathrm{bar}$ & $-7.0 \mathrm{kpa}$ & $-6.8 \mathrm{kpa}$ & $-5.5 \mathrm{kpa}$ \\
\hline $4.0 \mathrm{bar}$ & $-8.7 \mathrm{kpa}$ & $-8.4 \mathrm{kpa}$ & $-6.7 \mathrm{kpa}$ \\
\hline $4.5 \mathrm{bar}$ & $-10.9 \mathrm{kpa}$ & $-10.1 \mathrm{kpa}$ & $-7.8 \mathrm{kpa}$ \\
\hline $5.0 \mathrm{bar}$ & $-12.9 \mathrm{kpa}$ & $-12.2 \mathrm{kpa}$ & $-9.2 \mathrm{kpa}$ \\
\hline
\end{tabular}

Thus, the actual airflow velocity would be obviously lower than expected at higher supply air pressure. According to Bernoulli's equation, therefore, the vacuum gauge pressure at the center of the vacuum pad would be higher than expected because of the limited airflow velocity.

It is worth mentioning that the deviations between experimental and simulation results are inevitable. One most important reason is that the flow is assumed to be laminar flow and all initial and boundary conditions for CFD simulation are based on ideal assumptions. In the real flow field, however, turbulent flow as well as some time-varying parameters may exist, which are quite difficult to be modeled and numerically determined. Figure 16 shows an experimental example, where the generated lifting force makes successfully a CD-disk adhere to the developed vacuum pad. The supply pressure is adjusted to be 1 bar and the mass of the CD-disk is approximately $16 \mathrm{~g}$. 


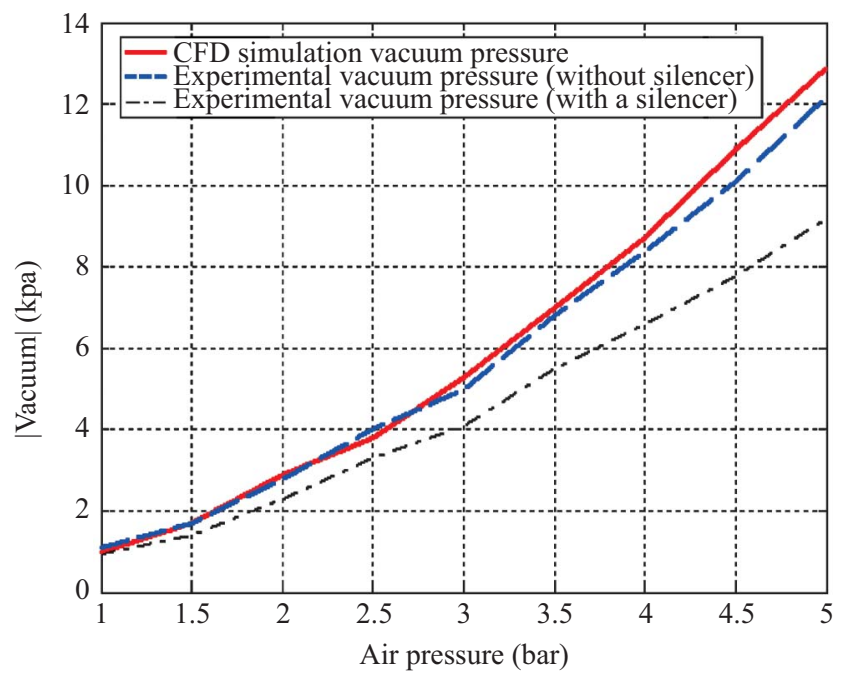

Fig. 15. Graphical comparison of the simulation and experimental vacuum pressure at the center of the vacuum pad.

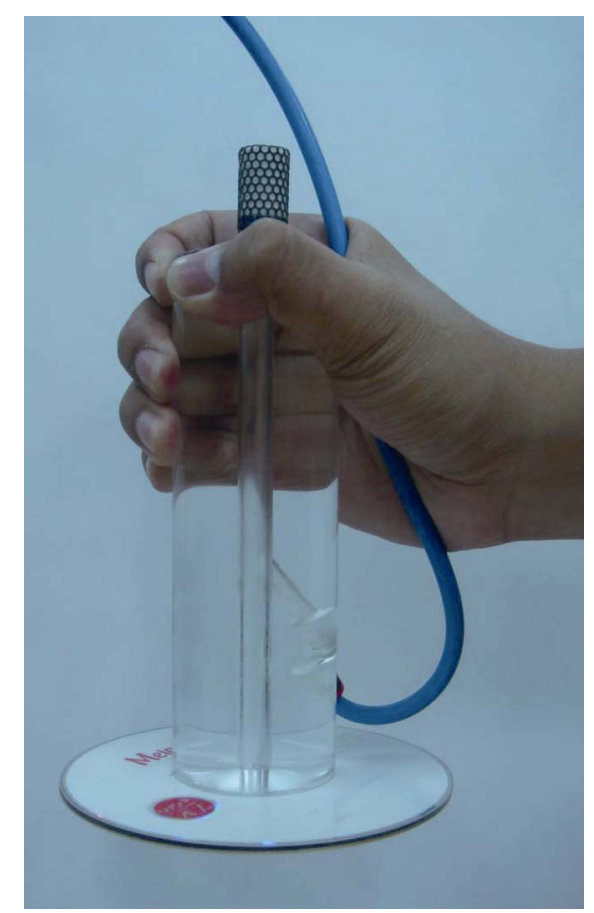

Fig. 16. An experimental example showing the developed vacuum pad with a lifted CD-disk.

\section{CONCLUSION}

In this paper, a novel, simple hand-held vacuum pad was successfully developed. After experimental tests, it is proved that the simple cylindrical vacuum pad can be easily grasped by the operator's hand and provide large lifting force output. Moreover, inexpensive manufacturing can also be achieved by replacing the complex nozzle with simple sudden reduction of pipe. In addition, three conclusions may be drawn from this research.
(1) The CFD-simulation is successfully applied to obtain the most suitable geometric dimension for the vacuum pad such that significant vacuum effect can be achieved.

(2) Though the developed hand-held vacuum pad without silencer can provide larger lifting force output, it is still recommended that the silencer should be installed to reduce the noise in real applications.

(3) Generally speaking, higher air supply pressure would give rise to larger lifting force output proportionally. However, it also contributes to higher air consumption. Therefore, the optimal adjustment of supply pressure depends chiefly on the demand of real application.

\section{ACKNOWLEDGMENTS}

The financial support of the National Science Council under grant number NSC-97-2622-E-224-006-CC3 is greatly appreciated.

\section{NOMENCLATURE}

\begin{tabular}{|c|c|c|}
\hline$A_{v}$ & {$\left[\mathrm{~m}^{2}\right]$} & effective area of pressure action \\
\hline$F_{\text {lift }}$ & {$[\mathrm{N}]$} & lifting force output \\
\hline$g$ & {$\left[\mathrm{~m} / \mathrm{s}^{2}\right]$} & gravity acceleration \\
\hline$P_{a t m}$ & {$[\mathrm{kPa}]$} & atmospheric gauge pressure \\
\hline$P_{1}$ & {$[\mathrm{kPa}]$} & pressure of fluid at station 1 \\
\hline$P_{2}$ & {$[\mathrm{kPa}]$} & pressure of fluid at station 2 \\
\hline$P_{v}$ & {$[\mathrm{kPa}]$} & $\begin{array}{l}\text { vacuum gauge pressure acting on the } \\
\text { work-piece inside the vacuum pad }\end{array}$ \\
\hline$W$ & {$\left[\mathrm{~N} / \mathrm{m}^{3}\right]$} & weight density of fluid \\
\hline$V_{1}$ & {$[\mathrm{~m} / \mathrm{s}]$} & velocity of fluid at station 1 \\
\hline$V_{2}$ & {$[\mathrm{~m} / \mathrm{s}]$} & velocity of fluid at station 2 \\
\hline$Z_{1}$ & {$[\mathrm{~m}]$} & elevation head at station 1 \\
\hline$Z_{2}$ & [m] & elevation head at station 2 \\
\hline
\end{tabular}

\section{REFERENCES}

1. Esposito, A., Fluid Power with Applications, 5th edition, Prentice-Hall, London, pp. 98-104 (2000).

2. Holbrook, A. E. K., "Design of rotary vacuum pumps for an automated manufacturing system," IEE Colloquium on Successful Cases of Integrated Product Design with Manufacturing Technology (Digest No: 1997/ 168), pp. 6/1 - 6/4 (1997).

3. Hsing Yang Technology Corp., Catalogue of Hsing Yang Technology Corp., available from (http://www.hsinyang.com.tw/twproduct.aspx\#h) (2006).

4. Huguet, D. and Codina, E., "CFD analysis of flow forces on direct acting relief mini-valves," Proceedings of the First International Conference on Computational Methods in Fluid Power Technology, Melbourne, Australia, pp. 201-210 (2003).

5. Kagawa, T., "New pneumatic techniques and applications," Proceedings of 7th JFPS International Symposium on Fluid Power, Toyama, Japan, Vol. 1, pp. 3-8 (2008).

6. Mark, H., "Adjustable vacuum valve," World Pumps, Elsevier Science, Vol. 2003, No. 446, pp. 3-48 (2003).

7. Renn, J. C., Chen, C. Y., and Lu, C. H., "Constant gap and minimum energy supply control for a proportional pneumatic vacuum pad," Proceedings of 7th JFPS International Symposium on Fluid Power, Toyama, 
Japan, Vol. 2, pp. 453-456 (2008).

8. Renn, J. C., Chen, C. Y., and Lu, C. H., "Gap control for a proportional floating vacuum pad," Proceedings of the Institution of Mechanical Engineers, Journal of Mechanical Engineering Science, Part C, Vol. 222, No. 11, pp. 2069-2072 (2008).

9. Renn, J. C. and Jan, D. Y., "Designing an energy-saving pneumatic vacuum pad for automated manufacturing system," Proceedings of the Institution of Mechanical Engineers, Journal of Mechanical Engineering Science, Part C, Vol. 221, No. 12, pp. 1679-1685 (2007).

10. Renn, J. C. and Wu, J. M., "Analysis and compensation of the flow force in a 4/3 oil hydraulic directional solenoid valve," Journal of the Chinese Society of Mechanical Engineers, Vol. 21, No. 2, pp. 209-215 (2000).
11. Ryuju Funayama, "Non-contact transfer components," General catalogue of air treatment auxiliary and vacuum KOGANEI Company, pp. 672-681 (2007).

12. Shen, S., Kishiyama, K., Parker, J. M., Wilson, N. G., and Schrage, D., "APT/LEDA RFQ vacuum pumping system," Proceedings of the Particle Accelerator Conference, Vol. 3, pp. 3625-3627 (1997).

13. SMC Corp., SMC-Best Pneumatics, Catalogue of SMC Corporation, Vol C003 (2000)

14. Vescovo, G. D. and Lippolis, A., "CFD analysis of flow forces on spool valves," Proceedings of the First International Conference on Computational Methods in Fluid Power Technology, Melbourne, Australia, pp. 315-331 (2003) 